\title{
SIGNED CHIP FIRING GAMES AND SYMMETRIC SANDPILE MODELS ON THE CYCLES *
}

\author{
Robert Cori ${ }^{1}$, Thi Ha Duong Phan ${ }^{2}$ \\ and Thi Thu Huong Tran ${ }^{2}$
}

\begin{abstract}
We investigate the Sandpile Model and Chip Firing Game and an extension of these models on cycle graphs. The extended model consists of allowing a negative number of chips at each vertex. We give the characterization of reachable configurations and of fixed points of each model. At the end, we give explicit formula for the number of their fixed points.
\end{abstract}

Mathematics Subject Classification. 05C57, 91A43, 68R15, $68 \mathrm{Rxx}$.

\section{INTRODUCTION}

The Chip Firing Game (CFG) was introduced by Björner, Lovász and Shor in 1991 [2]. A CFG is defined on an underlying directed graph $G=(V, E)$ and described by its configurations and the firing rule. Each configuration is a distribution of chips on $V$. The firing rule is that at each step, a vertex containing as many chips as its out-going degree gives one chip along each of its out-going edges. CFG is then showed having numerous applications in various fields, such as theoretical computer sciences, combinatorics, mathematics $[2,3,5,7,14]$.

The Sandpile Model (SPM) is introduced independently by Bak, Tang and Wiesenfied [1] to study a popular phenomena in physics called Self-Organized

\footnotetext{
Keywords and phrases. Sandpile Model, Chip Firing Game, circular distribution, Symmetric Sandpile Model, Signed Chip Firing Game, lattice structure.

* This paper was partially sponsored by the Vietnamese National Foundation for Science and Technology Development.

1 Labri, University Bordeaux 1, 33405 Talence Cedex, France. robert.cori@labri.fr

2 Institute of Mathematics, 18 Hoang Quoc Viet, Cau Giay district, Hanoi, Vietnam.

phanhaduong@math.ac.vn; ttthuong@math.ac.vn
} 
Criticality (SOC). It obeys the evolution rule that sandpiles with high energy collapse to sandpiles with lower energy to become more stable. The system then has been developed and broadened deeply in many different directions such as avalanche, cellular automata, discrete dynamical system [5,6,15]. Although these two systems (SPM and CFG) are introduced for different purposes at the beginning, they have actually a very close relation. Particularly, it is possible to code a SPM by a CFG on a linear graph [10]. So investigating CFG contributes to investigating SPM. Conversely, investigating SPM gives as well the results which are very specific and difficult to be done in CFG in general. For instance, it is proved that the system CFG on graphs with no closed component converges to an unique fixed point (i.e., configuration on which no rule can be applied) [14], but while the formula of fixed point of SPM was given explicitly [7], this is not yet the case for CFG.

One important research exploring the systems is to study all their configurations. More details, it comes up with the qualitative research on the convergence, on the characterizations of reachable configurations, of fixed point, and the various structures, such as order, lattice and algebra, of the configuration spaces, etc. Simultaneously, it also suggests the quantitative research on the complexity, the convergent time of the system. These problems are solved partly for the systems CFG and SPM on some particular graphs such as the line, the rectangular grids or even on general graphs.

In this paper, we study these problems for the systems SPM and CFG on cycle graphs. These problems also have a strong relation to the class of problems on cycles such as games of cards $[4,8,12]$. Furthermore, we are also interested in the signed versions of these systems, i.e., we allow the vertices to contain negative numbers of chips for CFG and the sandpiles to have negative heights for SPM. This also reflects deeply some natural phenomena: between sandpiles there may be holes (of negative heights), and besides the delivering chips from vertices containing many chips, it is dually possible receiving chips from vertices lacking (negative enough) chips [13].

The paper is organized as follows. In Section 2, we present some preliminary definitions and notations of SPMs and CFGs (signed and non-signed versions) on cycles and we then give the characterizations for their configurations. In Section 3, we point out the characterizations for their stable configurations and count them by giving explicit formulas.

\section{SPM, CFG, SSPM AND SCFG on CYClEs: DeFinitions AND NOTATIONS}

In this section, we present four models on cycles defined in term of discrete dynamical systems: Sandpile Model (SPM), Chip Firing Game (CFG) and their two generalizations called Symmetric Sandpile Model (SSPM) and signed chip firing game (SCFG). Then we show the close relationship between these systems. 
Let $C_{n}$ be a cycle graph of $n$ vertices $\{1,2, \ldots, n\} \quad(n \geq 3)$. Each sequence of integers $\left(a_{1}, a_{2}, \ldots, a_{n}\right)$ on vertices of $C_{n}$ is called circular distribution and we say that vertex $i$ contains $a_{i}$ chips (note that $a_{i}$ may be negative). We identify two circular distributions if they differ by a rotation of the cycle.

Definition 2.1. Let $k$ be a non-negative integer. The Sandpile Model on $C_{n}$ of weight $k$, denoted by $\operatorname{SPM}\left(C_{n}, k\right)$, is described as follows:

(i) The initial configuration is $(k, 0,0, \ldots, 0)$.

(ii) The evolution rule is the right rule as follows: a vertex gives one chip to its right neighbor vertex if it is at least 2 higher than this neighbor.

Definition 2.2. Let $k$ be a non-negative integer. The Symmetric Sandpile Model on $C_{n}$ of weight $k$, denoted by $\operatorname{SSPM}\left(C_{n}, k\right)$, is described as follows:

(i) The initial configuration is $(k, 0, \ldots, 0)$.

(ii) The evolution rule: addition to the right rule in SPM, there is also the left rule, that means a vertex gives one chip to its left neighbor vertex if it is at least 2 higher than this left neighbor.

Definition 2.3. Let $k$ be a non-negative integer. The Chip Firing Game on $C_{n}$ of weight $k$, denoted by $\operatorname{CFG}\left(C_{n}, k\right)$, is described as follows:

(i) The initial configuration is $(k, 0,0, \ldots, 0,-k)$.

(ii) The evolution rule is the positive rule as follows: a vertex containing at least 2 gives one chip to each of its two neighbors.

Definition 2.4. Let $k$ be a non-negative integer. The Signed Chip Firing Game on $C_{n}$ of weight $k$, denoted by $\operatorname{SCFG}\left(C_{n}, k\right)$, is described as follows:

(i) The initial configuration is $(k, 0, \ldots, 0,-k)$.

(ii) The evolution rule: addition to the positive rule in CFG, there is also the negative rule, that means a vertex containing at most -2 receives one chip from each of its neighbors.

\section{Notations.}

- We also denote by $\operatorname{SPM}\left(C_{n}, k\right), \operatorname{SSPM}\left(C_{n}, k\right), \operatorname{CFG}\left(C_{n}, k\right), \operatorname{SCFG}\left(C_{n}, k\right)$ the set of all reachable configurations, called configuration spaces, of the Sandpile Model, the Symmetric Sandpile Model, the Chip Firing Game and the Signed Chip Firing Game on $C_{n}$ of weight $k$ respectively.

- We then denote by $\operatorname{SPM}\left(C_{n}\right)$ the disjoint union of $\operatorname{SPM}\left(C_{n}, k\right)$ for all $k \geq 0$, and similarly for $\operatorname{SSPM}\left(C_{n}\right), \operatorname{CFG}\left(C_{n}\right), \operatorname{SCFG}\left(C_{n}\right)$.

- Let $a$ and $b$ be two circular distributions on $C_{n}$, we write $a \stackrel{(i, r)}{\longrightarrow} b$ (resp. $a \stackrel{(i, l)}{\longrightarrow} b$ ) if $b$ is obtained from $a$ by applying the right (resp. left) rule at vertex $i$; and $a \stackrel{(i,+)}{\longrightarrow} b$ (resp. $a \stackrel{(i,-)}{\longrightarrow} b)$ if $b$ is obtained from $a$ by applying the positive rule (resp. negative rule) at vertex $i$.

Figure 1 illustrates the configuration space of $\operatorname{SSPM}\left(C_{4}, 4\right)$ as an example. 


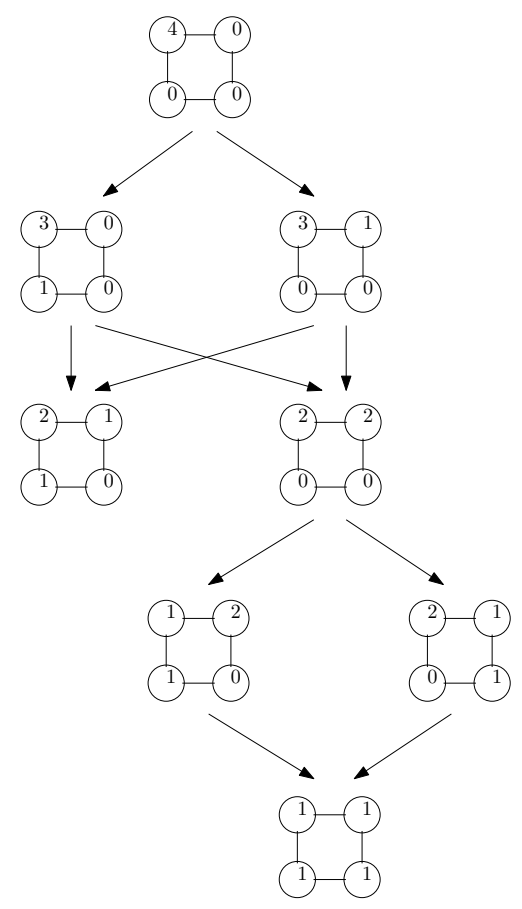

Figure 1. The space of configurations of $\operatorname{SSPM}\left(C_{4}, 4\right)$.

Remark 2.5. It is straightforward from the definitions that

(i) The configurations of $\operatorname{SPM}\left(C_{n}\right)$ and $\operatorname{SSPM}\left(C_{n}\right)$ are circular distributions of non-negative integers whereas the ones of $\mathrm{CFG}\left(C_{n}\right)$ and $\operatorname{SCFG}\left(C_{n}\right)$ are circular distributions of integers (may be negative).

(ii) We have the two following inclusions

$$
\operatorname{SPM}\left(C_{n}, k\right) \subseteq \operatorname{SSPM}\left(C_{n}, k\right) \text { and } \operatorname{CFG}\left(C_{n}, k\right) \subseteq \operatorname{SCFG}\left(C_{n}, k\right) .
$$

As mentioned above, on the line each SPM can be coded by a CFG. Studying the model SPM can give results on CFG and vice versa. However, we also perceive that the configurations of the $\operatorname{SPM}\left(C_{n}\right)$ (resp. $\left.\operatorname{SSPM}\left(C_{n}\right)\right)$ and $\operatorname{CFG}\left(C_{n}\right)$ (resp. $\left.\operatorname{SCFG}\left(C_{n}\right)\right)$ on the cycle are very different. For instance, given a circular distribution we can calculate the weight in the model SPM (which is equal to the sum of all its parts) whereas we even do not know exactly this quantity in the model $C F G\left(C_{n}\right)$. Nevertheless, next we prove that for each given weight they are in fact isomorphic.

Definition 2.6. Two discrete dynamical systems are called isomorphic if there exists a bijection between their configuration spaces and this bijection preserves their evolution rule. 
Let $a=\left(a_{1}, \ldots, a_{n}\right)$ be a circular distribution on $C_{n}$. We define

$$
d(a)=\left(a_{1}-a_{2}, \ldots, a_{n-1}-a_{n}, a_{n}-a_{1}\right) .
$$

It is straightforward that $d$ is a well-defined map from the set of circular distribution on $C_{n}$ to itself. Furthermore, we have the following

Proposition 2.7. Under the map d two systems $\operatorname{SPM}\left(C_{n}, k\right)$ and $C F G\left(C_{n}, k\right)$ are isomorphic; and two systems $\operatorname{SSPM}\left(C_{n}, k\right)$ and $S C F G\left(C_{n}, k\right)$ are isomorphic.

Proof. By the definition, $d(k, 0, \ldots, 0)=(k, 0, \ldots,-k)$ and so $d$ maps the initial configuration of $\operatorname{SPM}\left(C_{n}, k\right)$ (resp. $\operatorname{SSPM}\left(C_{n}, k\right)$ to the initial configuration of $\operatorname{CFG}\left(C_{n}, k\right)$ (resp. $\left.\operatorname{SCFG}\left(C_{n}, k\right)\right)$.

We prove that $d$ preserves the evolution rule between corresponding systems by showing that:

(i) $a \stackrel{(i, r)}{\longrightarrow} b$ if and only if $d(a) \stackrel{(i,+)}{\longrightarrow} d(b)$ (so that $d$ also preserves the rule of $\operatorname{SPM}\left(C_{n}, k\right)$ and $\left.\operatorname{CFG}\left(C_{n}, k\right)\right)$.

(ii) $a \stackrel{(i, l)}{\longrightarrow} b$ if and only if $d(a) \stackrel{(i,-)}{\longrightarrow} d(b)$.

Thus, $a \stackrel{(i, r)}{\longrightarrow} b$, then $a_{i}-a_{i+1} \geq 2$ and $d(a)_{i} \geq 2$. Hence, it is possible to apply the positive rule at vertex $i$ on $d(a)$ and obtain the configuration

$$
\left(d(a)_{1}, \ldots, d(a)_{i-1}+1, d(a)_{i}-2, d(a)_{i+1}+1, \ldots, d(a)_{n}\right) .
$$

On the other hand,

$$
b=\left(a_{1}, \ldots, a_{i}-1, a_{i+1}+1, \ldots, a_{n}\right)
$$

and

$$
d(b)=\left(d(a)_{1}, \ldots, d(a)_{i-1}+1, d(a)_{i}-2, d(a)_{i+1}+1, \ldots, d(a)_{n}\right) .
$$

Hence, $d(a) \stackrel{(i,+)}{\longrightarrow} d(b)$. Similarly for (ii) and so that $d$ preserves the evolution rule. So $d$ is isormorphic. Furthermore,

$$
d^{-1}(u)=\left(\alpha, \alpha-u_{1}, \alpha-u_{1}-u_{2}, \ldots, \alpha-u_{1}-\cdots-u_{n-1}\right),
$$

where $u=\left(u_{1}, \ldots, u_{n}\right) \in \operatorname{CFG}\left(C_{n}, k\right)\left(\right.$ resp. $\left.\operatorname{SCFG}\left(C_{n}, k\right)\right)$ and $\alpha=$ $\frac{k+\sum_{i=1}^{n-1}(n-i) u_{i}}{n}$.

It is remarkable that although $d$ is bijective from $\operatorname{SSPM}\left(C_{n}, k\right)$ (resp. $\operatorname{SPM}\left(C_{n}, k\right)$ ) to $\operatorname{SCFG}\left(C_{n}, k\right)$ (resp. $\operatorname{CFG}\left(C_{n}, k\right)$ ), it is not bijective from $\operatorname{SSPM}\left(C_{n}\right)$ (resp. $\operatorname{SPM}\left(C_{n}\right)$ ) to $\operatorname{SCFG}\left(C_{n}\right)$ (resp. $\operatorname{CFG}\left(C_{n}\right)$ ). Moreover, while $\operatorname{SSPM}\left(C_{n}, k\right)$ and $\left.\operatorname{SPM}\left(C_{n}, k\right)\right)$ are absolutely disjoint for different values $k$, $\operatorname{SCFG}\left(C_{n}, k\right)$ and $\operatorname{CFG}\left(C_{n}, k\right)$ may overlap each other, especially for values $k$ differing a multiple of $n$. So that a configuration of $\operatorname{SCFG}\left(C_{n}\right)$ may correspond to many configurations of $\operatorname{SSPM}\left(C_{n}\right)$ whose weights differ a multiple of $n$. 
On the other hand, for different values $k$, the systems evolve differently. The larger $k$ is, the more complicated the systems are. However, as far as we have known in many systems, their configurations are not so complicated. Particularly, we prove in Proposition 3.1 that for large enough values $k$ in the same residue class modulo $n$, the set of stable configurations of $\operatorname{SCFG}\left(C_{n}, k\right)\left(\operatorname{resp} \operatorname{CFG}\left(C_{n}, k\right)\right)$ coincide.

Next, we study a characterization for the configurations of the four systems. To do this we represent the characterization for the configurations of SPM on the line given by Goles and Kiwi [7]. Recall that the Sandpile Model on the line, denoted by $\operatorname{SPM}(k)$, is defined by its initial configuration $(k)$ and the right rule. We also denote by

$$
\mathrm{SPM}=\sqcup_{k=0}^{\infty} \operatorname{SPM}(k)
$$

the configuration space of the SPM on the line.

Let $a=\left(a_{1}, a_{2}, \ldots\right)$ be a sequence of positive integers. A pair $\left(a_{i}, a_{i+1}\right)$ is called a cliff (resp. plateau) of $a$ at position $i$ if $a_{i}-a_{i+1} \geq 2$ (resp. $\left.a_{i}-a_{i+1}=0\right)$.

Lemma 2.8 ([7]). A non-increasing sequence $\left(a_{1}, a_{2}, \ldots, a_{n}\right)$ of positive integers is a configuration of SPM on the line if between two consecutive plateaus there exists at least one cliff.

Theorem 2.9. Let a be a circular distribution on $C_{n}$. Then a is a configuration of $\operatorname{SPM}\left(C_{n}, k\right)$ if and only if there is a rotation vertices of $C_{n}$ such that a (in the sequence form) is a configuration of $\operatorname{SPM}(k)$ with the length at most $n$.

Proof. Let $a \in \operatorname{SPM}\left(C_{n}, k\right)$. Without loss of the generality, we assume that $a$ is reachable from $(k, 0, \ldots, 0)$ where $k$ is placed at the first vertex of $C_{n}$. Since only the right rule is applied, it creates the intermediate non-increasing sequences in the evolution to reach $a$. So vertex $n$ of $C_{n}$ never gives back to vertex 1 during the evolution. So $a$ is also a configuration of $\operatorname{SPM}(k)$. The converse is straightforward.

The following corollary is direct from Proposition 2.7 and Theorem 2.9.

Corollary 2.10. Let $a=\left(a_{1}, a_{2}, \ldots, a_{n}\right)$ be a circular distribution. Then $a$ is a configuration of $\operatorname{CFG}\left(C_{n}, k\right)$ if and only if $d^{-1}(a)$ is a configuration of $\operatorname{SPM}\left(C_{n}, k\right)$.

We recall that given a system we can define a 2 -ary relation $\preccurlyeq$ on its configuration space such that $a \preccurlyeq b$ if $a$ is reachable from $b$ by applying several steps of the evolution rule. This relation very depends on the evolution rule of the system and the distinct systems give the distinct relations. Furthermore, the relation $\preccurlyeq$ is in general not an ordered relation since a configuration can be reachable from itself and creating a cyclic in the orbit graph of its configuration space. However, in many investigated systems, the relation is an ordered relation. Particularly, the systems SPM and SSPM on the line investigated in $[6,11,15]$ together with the relation $\preccurlyeq$ on those are partially ordered sets (poset) and moreover the poset SPM 
forms a lattice in which any two its elements have a unique supremum and a unique infimum. Next we prove that $\operatorname{SPM}\left(C_{n}, k\right)$, and so $\operatorname{CFG}\left(C_{n}, k\right)$, also inherits the lattice structure of $\operatorname{SPM}(k)$.

Proposition 2.11. The poset $\left(\operatorname{SPM}\left(C_{n}, k\right), \preccurlyeq\right)\left(\operatorname{resp} . \quad\left(C F G\left(C_{n}, k\right), \preccurlyeq\right)\right)$ is a sub-lattice of the lattice $(\operatorname{SPM}(k), \preccurlyeq)(\operatorname{resp} .(C F G(k), \preccurlyeq))$.

Proof. By Proposition 2.7, it is sufficient to prove the statement for $\operatorname{SPM}\left(C_{n}, k\right)$. Let $a$ and $b$ be configurations of $\operatorname{SPM}\left(C_{n}, k\right)$. By Theorem 2.9, we assume that $a, b \in S P M(k)$ and $l(a), l(b) \leq n$. We prove that $c=\inf (a, b)$ and $d=\sup (a, b)$, where the supremum and infimum are taken in the lattice $S P M(k)$, are of the length at most $n$. We recall that if $u \preccurlyeq v$ in $S P M(k)$, i.e. $u$ is reachable from $v$ in $S P M(k)$, then $l(v) \leq l(u)$. So $l(d) \leq l(a) \leq n$ and $d \in S P M\left(C_{n}, k\right)$. The rest is implied from the explicit formulae of $\inf (a, b)$ in $S P M(k)([9])$. More precisely, we have $c=\left(c_{1}, c_{2}, \ldots, c_{l}\right)$, where $c_{i}$ is defined recursively as follows $c_{1}=\min \left\{a_{1}, b_{1}\right\}$ and $c_{i}=\min \left\{\sum_{j=1}^{i} a_{j}, \sum_{j=1}^{i} b_{j}\right\}-\sum_{j=1}^{i-1} c_{j}$. So that $l(c) \leq \max \{l(a), l(b)\} \leq n$ and $c \in S P M\left(C_{n}, k\right)$.

Figure 2 illustrates the lattice of $S P M(10)$ containing $S P M\left(C_{3}, 10\right)$ as a sub-lattice. The fixed point of $\operatorname{SPM}(10)$ is $(4,3,2,1)$ whereas the fixed point of $\operatorname{SPM}\left(C_{3}, 10\right)$ is $(4,3,3)$.

By some simple calculations, we get

Corollary 2.12. The unique fixed point of $S P M\left(C_{n}, k\right)$ is of the form

(i) $(p, p-1, \ldots, q, q, q-1, \ldots, 1,0, \ldots, 0)$ if $k \leq \frac{n(n-1)}{2}$, where

$$
p=\left[\frac{3+\sqrt{9+8 k}}{2}\right] \text { and } q=k-\frac{p(p+1)}{2} .
$$

(ii) $(p, p-1, \ldots, q+1, q, q, q-1, \ldots, p-n+3, p-n+2)$ if $k \geq \frac{n(n-1)}{2}+1$, where

$$
p=\left[\frac{2 k+n(n-2)}{2 n}+1\right] \text { and } q=k-\frac{(2 p-n+2)(n-1)}{2} \text {. }
$$

Here $[x]$ denotes the largest integer no greater than $x$.

Next, we give a characterization for the configurations of $\operatorname{SSPM}\left(C_{n}\right)$ as well as $\operatorname{SCF} G\left(C_{n}\right)$. To do this we first present the concept of 2-decomposition of the circular distributions which is more general than the concept of LR-decomposition (left-right decomposition) on the line $[6,15]$.

Definition 2.13. Let $a=\left(a_{1}, a_{2}, \ldots, a_{n}\right)$ be a circular distribution. Then $a$ is called 2-decomposable at $(i, j)$ (with $1 \leq i \leq j \leq n$ ) if $\left(a_{i-1}, a_{i-2}, \ldots, a_{1}, a_{n}, \ldots, a_{j+1}\right)$ and $\left(a_{i}, a_{i+1}, \ldots, a_{j}\right)$ are SPM configurations. Furthermore, $a$ is called 2-decomposable if there exist $i, j$ such that $a$ is 2-decomposable at $(i, j)$. 


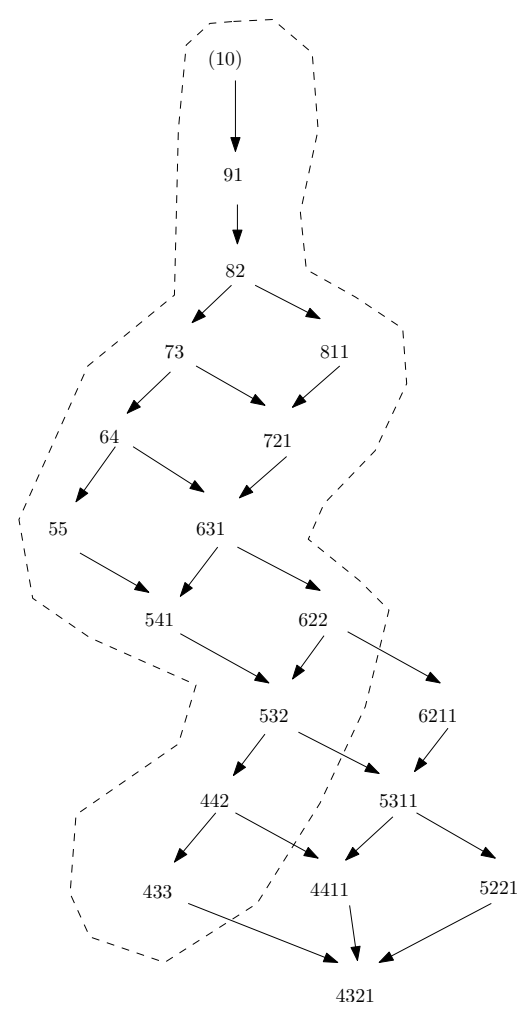

Figure 2. The sub-lattice $S P M\left(C_{3}, 10\right)$ in the lattice $S P M(10)$.

Remark 2.14. A 2-decomposable configuration a may be 2-decomposable at many $(i, j)$. For instance, $(2,5,5,4,1,1)$ is 2 -decomposable at $(1,2)$ and $(2,5)$ but not 2-decomposable at $(5,5)$. Furthermore, $(1,2,2,3,3,7,4,4,1)$ is not 2-decomposable.

Theorem 2.15. Let a be a circular distribution on $C_{n}$. Then a is a configuration of $\operatorname{SSPM}\left(C_{n}\right)$ if and only if $a$ is 2-decomposable.

Proof. To prove the inference indicator, by recurrence, we show that if $a$ is 2-decomposable at $(i, j)(1 \leq i \leq j \leq n)$ and $a$ evolves to $b$ by applying one step of the evolution rule then $b$ also is 2-decomposable. We only consider the following cases:
(i) $a \stackrel{(j, r)}{\longrightarrow} b$;
(ii) $a \stackrel{(i, l)}{\longrightarrow} b$;
(iii) $a \stackrel{(j+1, l)}{\longrightarrow} b$;
(iv) $a \stackrel{(i-1, r)}{\longrightarrow} b$. 
The others are deduced from the result in [7] saying that applying one step (or several steps) of the right rule on a SPM configuration (on the line) gives also a SPM configuration. So that if $a$ is 2-decomposable at $(i, j)$ then $b$ is also 2-decomposable at $(i, j)$.

We now prove the statement for four cases above. By Lemma 2.8, adding cliffs to or removing parts from a SPM configuration gives a SPM configuration. So if $a \stackrel{(j, r)}{\longrightarrow} b$, then $a_{j}-a_{j+1} \geq 2$ and $a$ is also 2-decomposable at $(i, j+1)$. According to this new decomposition of $a$ then $b$ is obtained from $a$ not by one of four above cases. Therefore, $b$ is 2-decomposable at $(i, j+1)$.

Similarly, $b$ is 2-decomposable at $(i+1, j)$ if (ii); $b$ is 2-decomposable at $(i, j-1)$ if (iii) and $b$ is 2-decomposable at $(i-1, j)$ if (iv).

Conversely, assume that $a$ is 2-decomposable at $(i, j)$ and that $a$ is of weight $k$. We need to show that $a$ is reachable from $(k)$ in $\operatorname{SSPM}\left(C_{n}, k\right)$. Put

$$
k_{1}=\sum_{t=i}^{j} a_{t} \text { and } k_{2}=\sum_{t=j+1}^{n} a_{t}+\sum_{t=1}^{i-1} a_{t},
$$

and so $k_{1}+k_{2}=k$.

Since $\left(a_{i}, \ldots, a_{j}\right)$ is a SPM configuration of weight $k_{1}$, so it is reachable from $\left(k_{1}\right)$ in SPM. Hence, $a$ is reachable from $\left(a_{1}, \ldots, a_{i-1}, k_{1}, 0, \ldots, 0, a_{j+1}, \ldots, a_{n}\right)$ by a sequence of applications the right rule. Similarly, $\left(a_{i-1}, \ldots, a_{1}, a_{n}, \ldots, a_{j+1}\right)$ is reachable from $\left(k_{2}\right)$ in SPM and, equivalently, $\left(a_{j+1}, a_{j+2}, \ldots, a_{n}, a_{1}, \ldots, a_{i-1}\right)$ is reachable from $\left(k_{2}\right)$ by a sequence of applications the left rule. Therefore, $a$ is reachable from $\left(0, \ldots, 0, k_{2}, k_{1}, 0, \ldots, 0\right)$ by a sequence of applications the rule of $\operatorname{SSPM}\left(C_{n}\right)$. And the latter configuration is reachable from $(0, \ldots, 0, k, 0, \ldots, 0)$ by a sequence of applications the rule of $\operatorname{SSPM}\left(C_{n}, k\right)$, where the column of height $k$ is at the position $i$ if $k_{1} \geq k_{2}$ and at the position $(i-1)$ if $k_{1}<k_{2}$.

The following is direct from Proposition 2.7 and Theorem 2.15.

Corollary 2.16. Let $u=\left(u_{1}, \ldots, u_{n}\right)$ be a circular distribution. Then $u$ is a configuration of $\operatorname{SCFG}\left(C_{n}, k\right)$ if and only if $d^{-1}(u)$ is 2-decomposable.

\section{Fixed points of $\operatorname{CFG}\left(C_{n}\right)$ And $\operatorname{SCFG}\left(C_{n}\right)$}

Although Corollary 2.16 gives a criteria for the configurations of $\operatorname{SCFG}\left(C_{n}\right)$, it requires us to calculate their inverse images by $d$ and then to check their 2-decomposability in $\operatorname{SSPM}\left(C_{n}\right)$. In this section, we present a simple and direct characterization for the fixed points (not all their configurations) of $\operatorname{SCFG}\left(C_{n}\right)$. Based on this characterization, we give an enumeration for these fixed points. We first classify the configurations of $\operatorname{CFG}\left(C_{n}\right)$ and those of $\operatorname{SCFG}\left(C_{n}\right)$. 
Proposition 3.1. Let $k, l$ be non-negative integers. Then,

(i) If $k \neq l \bmod n$ then

$$
C F G\left(C_{n}, k\right) \cap C F G\left(C_{n}, l\right)=\emptyset
$$

and

$$
\operatorname{SCFG}\left(C_{n}, k\right) \cap \operatorname{SCFG}\left(C_{n}, l\right)=\emptyset .
$$

Consequently, the intersection of the set of fixed points of $\operatorname{SCFG}\left(C_{n}, k\right)$ and those of $\operatorname{SCFG}\left(C_{n}, l\right)$ is empty.

(ii) If $k=l \bmod n$ and $k, l \geq\left[\frac{n+1}{2}\right]^{2}$ then the set of fixed points of $C F G\left(C_{n}, k\right)$ (resp. $\left.\operatorname{SCF} G\left(C_{n}, k\right)\right)$ is equal to those of $\operatorname{CFG}\left(C_{n}, l\right)$ (resp. $\left.\operatorname{SCFG}\left(C_{n}, l\right)\right)$.

Proof.

(i) We prove that if $u=\left(u_{1}, u_{2}, \ldots, u_{n}\right) \in \operatorname{SCFG}\left(C_{n}, k\right)$, then

$$
\sum_{i=1}^{n-1} i u_{i}=k \quad \bmod n
$$

by showing that if $u \stackrel{(i,+)}{\longrightarrow} v$ (similarly, $u \stackrel{(i,-)}{\longrightarrow} v)$ then

$$
\sum_{t=1}^{n-1}(n-t) u_{t}=\sum_{t=1}^{n-1}(n-t) v_{t} \bmod n .
$$

Recall that

$$
v=\left(u_{1}, \ldots, u_{i-1}+1, u_{i}-2, u_{i+1}+1, \ldots, u_{n}\right) .
$$

By simple calculations, the expression $\left(^{*}\right)$ is deduced easily from the fact that

$$
\sum_{t=1}^{n-1} t u_{t}=\sum_{t=1}^{n-1} t v_{t} \text { for } i=1,2, \ldots, n-2,
$$

and

and

$$
\sum_{t=1}^{n-1} t u_{t}=\sum_{t=1}^{n-1} t v_{t}+n \text { for } i=n-1
$$

$$
\sum_{t=1}^{n-1} t u_{t}=\sum_{t=1}^{n-1} t v_{t}-n \text { for } i=n .
$$

(ii) Let $u$ be a fixed point of $\operatorname{SCFG}\left(C_{n}, k\right)$. By Proposition 2.7 and Theorem 2.15, $d^{-1}(u)$ is a fixed point of $\operatorname{SSPM}\left(C_{n}, k\right)$ and 2-decomposable at $(i, j) \quad(1 \leq i \leq$ $j \leq n)$. Therefore, the configuration $d^{-1}(u)+1$, which is obtained from $d^{-1}(u)$ by adding 1 to each its part, is also 2-decomposable at $(i, j)$ and on which neither the right nor the left rule can be applied. So $d^{-1}(u)+1$ is a fixed point of $\operatorname{SSPM}\left(C_{n}, n+\right.$ $k)$. Furthermore, $d\left(d^{-1}(u)+1\right)=u$. Hence, $u$ is a fixed point of $\operatorname{SCFG}\left(C_{n}, n+k\right)$. Conversely, let $u$ be a fixed point of $\operatorname{SCFG}\left(C_{n}, k+n\right)$. Similarly, $d^{-1}(u)-1$ contains the non-negative parts (since $k \geq\left[\frac{n+1}{2}\right]^{2}$ ) and is a fixed point of $\operatorname{SSPM}\left(C_{n}, k\right)$. Hence, $d\left(d^{-1}(u)-1\right)=u$ is also a fixed point of $\operatorname{SCFG}\left(C_{n}, k\right)$. 
As we remarked in the previous section that for large enough values of $k$ in a residue class modulo $n$, although the set of fixed points of $\operatorname{SSPM}\left(C_{n}, k\right)$ (resp. $\left.\operatorname{SPM}\left(C_{n}, k\right)\right)$ are disjointed, the heights of their columns differ up-to a constant. In the other word, if $\left(a_{1}, \ldots, a_{n}\right)$ is a fixed point of $\operatorname{SSPM}\left(C_{n}, k\right)\left(\operatorname{resp} \operatorname{SPM}\left(C_{n}, k\right)\right)$, then $\left(a_{1}+1, \ldots, a_{n}+1\right)$ is a fixed point of $\operatorname{SSPM}\left(C_{n}, k+n\right)\left(\operatorname{resp} \operatorname{SPM}\left(C_{n}, k+n\right)\right)$ (Prop. 3.1). And so that their images by $d$ in $\operatorname{SCFG}\left(C_{n}, k\right)\left(\operatorname{resp} \operatorname{CFG}\left(C_{n}, k\right)\right)$ and in $\operatorname{SCFG}\left(C_{n}, k+n\right)$ (resp. $\operatorname{CFG}\left(C_{n}, k+n\right)$ ) coincide.

By Corollary 2.12, $\operatorname{CFG}\left(C_{n}, k\right)$ has a unique fixed point whereas $\operatorname{SCFG}\left(C_{n}, k\right)$ may have many fixed points. By Proposition 3.1(ii), the set of fixed points of $\operatorname{SCFG}\left(C_{n}\right)$ includes the fixed points of $\operatorname{SCFG}\left(C_{n}, k\right)$ for small values of $k$ and the $n$ distinct residue classes of fixed points of $\operatorname{SCFG}\left(C_{n}, k\right)$ for large values of $k$. For the small $k$, their fixed points can be found directly by taking the inverse images of $d$ of 2-decomposable fixed points. We next characterize and enumerate the fixed points of $\operatorname{SCFG}\left(C_{n}\right)$ for all $k \geq\left[\frac{n+1}{2}\right]^{2}$.

For convenience, we denote by $F P\left(\operatorname{SCFG}\left(C_{n}, k\right)\right.$ the set of fixed points of $\operatorname{SCFG}\left(C_{n}, k\right)$ and

$$
F P\left(\operatorname{SCFG}\left(C_{n}\right)\right)=\bigcup_{k \geq\left[\frac{n+1}{2}\right]^{2}} F P\left(\operatorname{SCFG}\left(C_{n}, k\right)\right)
$$

Recall that each fixed point of $\operatorname{SCFG}\left(C_{n}\right)$ is a circular distribution on $C_{n}$ and its chips at vertices are $0,1,-1$. By a rotation, next we can consider $F P\left(\operatorname{SCFG}\left(C_{n}\right)\right)$ as words on the alphabet $\{0,1, \overline{1}\}$ where the letter $\overline{1}$ is understood as -1 .

Theorem 3.2. The set $F P\left(S C F G\left(C_{n}\right)\right)$ is determined as follows

(1) $F P\left(S C F G\left(C_{3}\right)\right)=\{(000) ;(10 \overline{1}) ;(1 \overline{1} 0)\}$.

(2) $F P\left(S C F G\left(C_{4}\right)\right)=\{(0000) ;(1 \overline{1} 00) ;(10 \overline{1} 0) ;(100 \overline{1}) ;(11 \overline{1} \overline{1})\}$.

(3) $\operatorname{FP}\left(S C F G\left(C_{n}\right)\right)$, with $n \geq 5$, includes the words $w$ on the alphabet $\{0,1, \overline{1}\}$ satisfying the following properties:

(i) $w$ starts from 1 ;

(ii) in $w$, the number of occurrences of 1 is equal to that of $\overline{1}$;

(iii) $w$ avoids the subsequences: $\overline{1} 1,1001, \overline{1} 00 \overline{1}$ and 00000 ;

(iv) If $w$ has 4 occurrences of 0 then it must end by 0 and does not contain the subword $1 \overline{1}$.

Proof.

(1) It is straightforward from the fact that all 2-decomposable fixed points of $\operatorname{SSPM}\left(C_{3}\right)$ are of the form $(a, a, a) ;(a+1, a, a)$ and $(a+1, a, a)$.

(2) It is straightforward from the fact that all 2-decomposable fixed points of $\operatorname{SSPM}\left(C_{4}\right)$ are $(a, a, a, a) ;(a+1, a, a+1, a+1) ;(a+1, a, a, a+1) ;(a+1, a, a, a)$ and $(a+1, a, a-1, a)$.

(3) Let $w \in F P\left(S C F G\left(C_{n}\right)\right)$. Since the sum of the parts of $w$ is equal to 0 , the statement $(\mathrm{ii})$ is obvious. By a rotation, we assume that $w$ avoids the subsequence 11 . By Theorem 2.15, $d^{-1}(w)$, taken in $\operatorname{SSPM}\left(C_{n}, k\right)$ for $k \geq\left[\frac{n+1}{2}\right]^{2}$, 
is 2-decomposable. So each its decomposed part does not contain more than one plateau corresponding to one occurrence of 0 and the subsequences 1001 and $\overline{1} 00 \overline{1}$ are forbidden in $w$. Furthermore, we may allow to have at most two plateaus at two splitting positions. Therefore, we have at most 4 plateaus in $d^{-1}(w)$ corresponding 4 occurrences of $0 s$ and so the subsequence 00000 is forbidden in $w$. Moreover, for $n \geq 5, w$ contains at least 1 occurrence of 1 and we can assume $w$ starts from 1 , avoids the subsequence $\overline{1} 1$. The statements (i) and (iii) are so satisfied. To prove (iv) we remark that if $w$ contains exactly 4 occurrences of $0 s$ then $d^{-1}(w)$ must have two plateaus at two splitting positions. Rotating $w$ such that (i) and (iii) are satisfied says that the two plateaus at two splitting positions will give one occurrence of 0 at the end and one occurrence of 0 between the last 1 and the first $\overline{1}$ of $w$. So (iv) is satisfied.

We now give an explicit formula for the number of fixed points of $\operatorname{SCFG}\left(C_{n}\right)$ by counting words in the above theorem.

Theorem 3.3. The cardinality of $F P\left(S C F G\left(C_{n}\right)\right)$ is

(i) 3 if $n=3$;

(ii) 5 if $n=4$;

(iii) $\frac{(n-1)^{2}}{2}$ if $n$ is odd and $n \geq 5$;

(iv) $\frac{n(n-2)}{2}$ if $n$ is even and $n \geq 6$.

Proof. It is sufficient to prove (iii) and (iv). To do this, by Theorem 3.2 we count the number of ways to insert some 0 s into a sequence including the $1 \mathrm{~s}$ before the 1s such that the conditions of Theorem 3.2(3.2) are satisfied.

(iii) $n=2 l+1$ : Let $w \in \mathcal{L}(S C F G)$ then $w$ has either one or three occurrences of 0 (by 3.2 (ii) of Theorem 3.2).

(a) $w$ has one occurrence of 0 . Then this 0 may appear at any position except for the first position (since $w$ starts from 1 ). So we have $n-1$ such words $w$.

(b) $w$ has 3 occurrences of $0 s$. Then $w$ has $(l-1)$ occurrences of 1 and so that of $\overline{1}$. Denote by $A$ the set of words of $(l-1)$ occurrences of $1 s$; of $(l-1)$ occurrences of $\overline{1} s$ and 3 occurrences of $0 s$ satisfying the conditions $3.2(\mathrm{i}), 3.2(\mathrm{ii})$ in Theorem 3.2 and avoiding the subsequence $\overline{1}$. Denote by $B$ the set of words in $A$ not satisfying all conditions of Theorem 3.2(3.2). Then $|A|$ is equal to the number of ways to choose 3 positions for 0 s from $n-1$ positions except for the first position. Hence, $|A|=C_{n-1}^{3}$.

On the other hand, the words of $B$ must contain the subsequence either 1001 or $\overline{1} 00 \overline{1}$. The number of words of $B$ containing the subsequence 1001 (resp. $\overline{1} 00 \overline{1})$ and not containing the subsequence 10001 (resp. $\overline{1} 000 \overline{1})$ is $(n-l-1) C_{l-1}^{2}$. Here, we have $C_{l-1}^{2}$ ways to choose 2 positions of 0 from $(l-1)$ its possible positions such that 1001 (resp. $\overline{1} 00 \overline{1})$ is its the subsequence; and $(n-l-1)$ ways to choose the rest 0 . Similarly, the number of words of $B$ containing the 
subsequence 10001 (resp. $\overline{1} 000 \overline{1}$ ) is $C_{l}^{3}$. Hence,

$$
|B|=2(n-l-1) C_{l-1}^{2}+2 C_{l}^{3} .
$$

Therefore,

$$
\left|F P\left(\operatorname{SCFG}\left(C_{n}\right)\right)\right|=(n-1)+(|A|-|B|)=\frac{(n-1)^{2}}{2} .
$$

(iv) $n=2 l$ : Let $w \in \mathcal{L}\left(\operatorname{SCFG}\left(C_{n}\right)\right)$. $w$ has no or 2 or 4 occurrences of 0 .

(a) if $w$ has no occurrence of 0 then $w=1 \ldots 1 \overline{1} \ldots \overline{1}$ and we have a unique $w$.

(b) if $w$ has exactly 2 occurrences of 0 s then it has $(l-1)$ occurrences of 1 and also $(l-1)$ occurrences of $\overline{1}$ s. So that the number of such words $w$ is $C_{n-1}^{2}-$ $2 C_{l-1}^{2}$. Here, $C_{n-1}^{2}$ is the number of words of 2 occurrences of 0 s satisfying the conditions $3.2(\mathrm{i})$ and $3.2(\mathrm{ii})$ of Theorem 3.2 and avoiding the subsequence $\overline{1} 1$; and $C_{l-1}^{2}$ is the number of words satisfying the conditions $3.2(\mathrm{i})$ and $3.2(\mathrm{ii})$ of Theorem 3.2, avoiding the subsequence $\overline{1} 1$ but containing the subsequence 1001 (resp. $\overline{1} 00 \overline{1})$.

(c) if $w$ has 4 occurrences of 0 s then it has $(l-2)$ occurrences of 1 and also $(l-2)$ occurrences of $\overline{1} s$. By 3.2(iv) of Theorem 3.2, w ends by 0 and has at least 1 occurrence of 0 between the last 1 and the first $\overline{1}$.

- $w$ is of the form $(1 \ldots 0 \ldots 10 \overline{1} \ldots \overline{1} 0)$ that means the first 0 is between two 1 s. We have $(l-3)$ ways to choose the first $0 ; 1$ way for the second 0 (as just after the last 1$)$; and $(l-1)$ ways for the third 0 at any positions after the second 0 ; and 1 way for the last 0 at the end of $w$. So we have $(l-1)(l-3)$ such words $w$.

- $w$ is of the form $(1 \ldots 100 \overline{1} \ldots \overline{1} 0)$ that means the first two 0s are between the first 1 and the last $\overline{1}$. So we have $(l-1)$ ways to choose the third 0 at any positions after the first two 0 s and we have $(l-1)$ such words $w$.

- $w$ is of the form $(1 \ldots 10 \overline{1} \ldots 0 \ldots \overline{1} 00)$ that means the first 0 is at the between the last 1 and the first $\overline{1}$, the second 0 is between two $\overline{1} s$. So we have $(l-3)$ ways to choose the second 0 and hence we have $(l-3)$ such words $w$.

- $w$ if of the form $(1 \ldots 10 \overline{1} \ldots \overline{1} 000)$ that means the first 0 is between the last 1 and the first $\overline{1}$ and the three last 0 s are at the end. So we have a unique such word $w$.

Taking the sum of these values we obtain $l(l-2)$ words of $w$ in this case. Hence,

$$
\left|F P\left(\operatorname{SCFG}\left(C_{n}\right)\right)\right|=1+C_{n-1}^{2}-2 C_{l-1}^{2}+l(l-2)=\frac{n(n-2)}{2} .
$$

\section{REFERENCES}

[1] P. Bak, C. Tang and K. Wiesenfeld, Self-organized criticality: An explanation of 1/f noise. Phys. Rev. Lett. 59 (1987) 381-284.

[2] A. Björner, L. Lovász and P.W. Shor, Chip-firing games on graphs. Eur. J. Combin. 12 (1991) 283-291. 
[3] R. Cori and D. Rossin, On the sandpile group of dual graphs. Eur. J. Combin. 21 (2000) $447-459$.

[4] J. Desel, E. Kindler, T. Vesper and R. Walter, A simplified proof for the self-stabilizing protocol: A game of cards. Inf. Process. Lett. 54 (1995) 327-328.

[5] D. Dhar, P. Ruelle, S. Sen and D.-N. Verma, Algebraic aspects of abelian sandpile models. J. Phys. A 28 (1995) 805-831.

[6] E. Formenti, B. Masson and T. Pisokas, Advances in symmetric sandpiles. Fundam. Inf. 76 (2007) 91-112.

[7] E. Goles and M.A. Kiwi, Games on line graphes and sand piles. Theoret. Comput. Sci. 115 (1993) 321-349.

[8] E. Goles, M. Morvan and H.D. Phan, Lattice structure and convergence of a game of cards. Ann. Combin. 6 (2002) 327-335.

[9] E. Goles, M. Morvan and H.D. Phan. Sandpiles and order structure of integer partitions. Discrete Appl. Math. 117 (2002) 51-64.

[10] E. Goles, M. Morvan and H.D. Phan, The structure of linear chip firing game and related models. Theoret. Comput. Sci. 270 (2002) 827-841.

[11] É. Goles and M. Latapy, Clémence Magnien, Michel Morvan and Ha Duong Phan. Sandpile models and lattices: a comprehensive survey. Theoret. Comput. Sci. 322 (2004) 383-407.

[12] S.-T. Huang. Leader election in uniform rings. ACM Trans. Program. Lang. Syst. 15 (1993) 563-573.

[13] R. Karmakar and S.S. Manna, Particle hole symmetry in a sandpile model, J. Stat. Mech. 2005 (2005) L01002.

[14] M. Latapy and H.D. Phan, The lattice structure of chip firing games. Physica D 115 (2001) $69-82$.

[15] H.D. Phan, Two sided sand piles model and unimodal sequences. RAIRO - Theor. Inf. Appl. 42 (2008) 631-646.

Communicated by B. Vallée.

Received March 20, 2012. Accepted August 7, 2012. 